\title{
Medici e pazienti sul web
}

\author{
Alessandra Lanzo', Giuseppe Quintaliani², Anna Colaci ${ }^{3}$ \\ ${ }^{1}$ Dr.ssa in Scienze della Comunicazione, Lecce \\ ${ }^{2}$ Coeditor Giornale Tecniche Nefrologiche \& Dialitiche, Perugia \\ ${ }^{3}$ Professore Associato Storia della Pedagogia Università Salento, Lecce
}

\begin{abstract}
Patients, Doctors, And The internet
Abstract. Web 2.0 and its evolution have changed the way we interact. They have revolutionized information, communication and training in medicine. Also the medicine becomes 2.0. On the one hand the patient empowerment and a more shared and human medicine, on the other the scientific community has access to a continuous information. The communication 2.0 and its tools allow the healthcare world to share medical information with followers, to store and manage the useful articles in an efficient manner, to be always up to date and to teach patients with a greater chance to establish an empathic and dynamic relationship. The evolution of the web and its tools represent steps towards an ideal medicine.
\end{abstract}

Key words: Web 2.0, Blog, Facebook, Management, Health 2.0, Social media, Social network, Twitter, Web 2.0, Youtube

Conflict of interest: None.

Financial support: None.

Accettato: 28 Maggio 2014

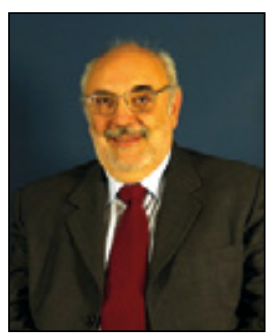

Giuseppe Quintaliani

\section{Introduzione}

L'ambiente comunicativo in cui interagiamo è puntualmente ridefinito dalle possibilità che $\mathrm{i}$ moderni modelli di comunicazione ci offrono.

L'avvento del web 2.0 e la sua continua evoluzione spalancano le porte a forme del vivere sociale che ci offrono una dinamicità di percorsi autoformativi e che sono costruttivi di nuove organizzazioni simboliche, le quali migliorano i nostri parametri cognitivi. Siamo tutti alle prese con un'informazione e una comunicazione partecipativa e collaborativa. Anche la scienza medica non sfugge alla democratizzazione della conoscenza. La medicina si fa 2.0: da un lato della scrivania l'empowerment del paziente, dall'altro il professionista che ha accesso a una formazione continua da qualsiasi luogo in qualsiasi momento. Tra entusiasmo e scetticismo i medici abbandonano i corsi di aggiornamento e i ritagli dei trafiletti delle riviste per attingere dalla rete attraverso editori virtuali. Il medico volgarizza il proprio sapere e impara ad apprezzare e ad attingere anche dall'associazionismo in rete dei pazienti e, dunque, dalla conoscenza che si genera nella condivisione di vissuti di malattie e farmaci, arricchendo, in questo modo, l'informazione scientifica. Il paziente considera, oggi, la salute sempre più un servizio, pertanto sente proprio il diritto di conoscere, valuta- re, paragonare e giudicare, sviluppando una consapevolezza della propria malattia. Sono i trend del Censit del due Ottobre del 2012 ad attestare l'empowerment del paziente. Il paziente, con l'associazionismo in rete, le cui pagine web risultano per gran parte affidabili e riccamente educative, comunica orizzontalmente e lateralmente. Egli rompe con la tradizionale e asimmetrica relazione medico-paziente, fondata su un'autoreferenzialità dell'esperto e con la dipendenza da quest'ultimo. I loro scambi generano un sistema di conoscenze che cresce parallelamente, indubbiamente diverso da quello scientifico, ma sicuramente più umano. L'evoluzione del web sostanzia di umanità la medicina, quasi dando spazio alle rivendicazioni della medicina narrativa.

Il presupposto secondo cui una relazione medico-paziente dinamica ed empatica, mirata alla comprensione reciproca, risulta terapeutica mette in evidenza come le opportunità di comunicazione e informazione del 2.0 e dei suoi strumenti possano promuovere maggiormente una relazione di questo tipo.

\section{La comunicazione via web}

I nuovi tool di una comunicazione aggregativa sono i wiki, $i$ podcast, le On-line Health Community, i social network, i blog e i feed RSS. Wiki significa veloce. I wiki, oggi, sono strumenti indispensabili di creazione e condivisione di informazione anche per gli utenti inesperti, i quali possono con grande facilità e velocità creare il proprio sito $w e b$ con pagine collegate 
fra loro, modificandone il contenuto in qualsiasi momento. Rappresentano la possibilità di interazione sociale attiva e veloce fra chi condivide uno stesso interesse, sfruttando il modello di comunicazione molti a molti.

La testimonianza di wiki per eccellenza è "Wikipedia" che, nonostante sia una libera enciclopedia on-line generalista, è la più completa anche in ambito medico-scientifico, che risulta affidabile e con un'altissima percentuale di veridicità anche in confronto con il colosso inglese "Nature".

Il "New England Journal of Medicine" è stato il primo a distribuire contenuti audio e diapositive dei propri articoli attraverso la tecnologia podcast, ossia un file audio o video che è possibile scaricare dalla rete e ascoltare attraverso un lettore multimediale, fruibile da chiunque, in qualsiasi momento e, soprattutto, in modalità offline, presupposto che lo ha reso mezzo privilegiato di trasmissione di contenuti multimediali. $\mathrm{E}$, ancora, società e riviste scientifiche inviano il sommario del proprio numero in uscita attraverso la tecnologia del feed $R S S$, un servizio che indirizza e organizza automaticamente i contenuti del sito web d'origine in un'area apposita dell'utente, da cui possono essere letti senza il collegamento al sito web in questione.

L'incontro virtuale tra i professionisti, tra professionisti e formazione professionale e tra cittadini/pazienti avviene nelle moderne piattaforme dei blog e dei social network, i primi più antichi e superati ormai dai secondi per un maggiore grado di libertà offerto agli utenti. Questi hanno rivoluzionato la formazione, l'informazione e la comunicazione in medicina. Da annoverare è l'esperienza di "Sermo", dove più di centoventimila medici americani si confrontano su qualsiasi sfera medica, e, ancora, "Doc2Doc", "Cardioexchange", nel caso italiano "Doctor33" e "Dottnet" e "Renalgate". L'esperienza di quest'ultimo sito è interessante; infatti non è solo un sito $w e b$ così come lo si intendeva, ma è diventato un vero e proprio network con il blog (http://renalgate.wordpress.com/), la pagina Facebook (https://www.facebook.com/), l'account Twitter (https://twitter.com/Pqr9ap) e linkedln (https://www. linkedln.com/hp/?dnr=t) e il canale video (https://www.youtube.com/user/renalgate\#g/f), tutti integrati tra loro.

Fra i social network dedicati, esperienze significative, impensabili fino a poco tempo fa, sono le comunità ibride e le comunità di pazienti per pazienti. Dove il medico incontra il paziente, il paziente può domandare e il medico può sintetizzare gli aggiornamenti e le ricerche, per esempio, rendendole più accessibili; sono realtà meno formali con uno stile comunicativo più facile e immediato per l'utente non competente, realtà utili ai fini di una comunicazione medico-paziente orientata alla comprensione e dunque a una relazione terapeutica efficace ("Medhelp", "Medgle", "Curetogether"); esistono realtà dove il paziente, invece, racconta il proprio vissuto agli atri pazienti, le On-line Health Community, e dove il cittadino ha la possibilità di condivisione ed espressione, la possibilità di informare e informarsi e di essere più consapevole, accedendo a una fonte di risorse conoscitive incredibile per chi si trova davanti a una scelta di salute. Se da un lato questa realtà risulta utile per la mole di informazioni interessanti, dall'altra risulta terapeutica: l'appartenenza a una comunità, la condivisione e il racconto del proprio sé hanno scientificamente riscon- tri positivi sul rapporto che il malato instaura con la propria malattia e con la necessità di ridefinire la propria identità sociale in seguito alla condizione diversa in cui il paziente si ritrova a vivere, soprattutto nei casi di malattia invalidante o cronica ("Patienlikeme", "Mdjunction", "Carepages"). Si parla oggi spesso di blogterapia, ma se la cybermedicina si fa spazio sui social network dedicati, i social generalisti sono tempestati da pagine e canali di contenuto medico-scientifico, aperti da società scientifiche, riviste e organizzazioni sanitarie che promuovono e rilanciano i contenuti e gli aggiornamenti dei propri siti web sui social network (Facebook, Twitter, Youtube) che spopolano. Sono la loro forza aggregativa e la loro potenzialità nel diffondere $\mathrm{i}$ contenuti in maniera virale $\mathrm{a}$ renderli attraenti.

Internet, allora, si inserisce nel quadro globale della sanità come la possibilità di una rete relazionale in cui si sperimenta uno sbocco verso una medicina più chiara, più completa, solidale e umana, come un luogo di scambio, sostegno, guida ed educazione condivisa. I progressi della telemedicina insieme alle opportunità offerte dall'evoluzione del web rappresentano piccoli passi verso una sanità ideale. È in atto un cambio di episteme, una di quelle transizioni che cambiano il modo di essere e di vedere la sanità, la quale deve anche, però, fronteggiarne i rischi. È necessario tenere in considerazione, oltre alle opportunità, anche alcune criticità del web e del conseguente concetto di webautonomy e information overlap (sovraccarico di informazione): la questione della privacy sulla rete e della violazione dei dati sensibili degli internauti è un tema caldo, dalla normativa ancora poco chiara; la democratizzazione del sapere apre le porte al dilettantismo e ad altri imprevisti circa l'affidabilità e la veridicità dei contenuti; e, ancora, il sovraccarico di informazioni e l'incapacità di discriminare l'informazione più attendibile può condurre all'errore di conferma, confirmation bias, ossia alla scelta delle informazioni più facili, e alla condizione, nel peggiore dei casi, di cyberchondria.

\section{Come può essere utile il web 2.0 in sanità?}

Perché il mondo scientifico utilizza gli strumenti del web 2.0: a) diffondere l'informazione tra i "follower"

b) archiviare alcuni articoli utili

c) permettere a tutti di seguire conferenze e presentazioni in differita

d) insegnare ai pazienti e/o informarli

e) tenersi aggiornati continuamente

f) formule e calcolatori.

La caratteristica più saliente del web 2.0 è la possibilità di partecipare attivamente alle piattaforme di comunicazione online (commenti, critiche, suggerimenti), non rendendo palese a tutti la propria posizione, ma comunque interagendo non solo con l'estensore dell'articolo o del post, ma con tutti coloro che sono in grado di interagire con l'informazione stessa. Analizziamo più da vicino le varie possibilità:

a) diffondere l'informazione che ci interessa tra i "follower" È possibile, una volta che l'esperto seleziona l'informazione, renderla disponibile immediatamente a tutti coloro che se- 
guono la sua pagina sui social network. È esperienza comune che, a partire da un blog, sia possibile alimentare sia Facebook che Twitter. Ed è possibile ancora, una volta selezionata un'informazione (supponiamo che un collega chieda a un altro il trattamento in urgenza dell'iperpotassiemia) (1), trasmetterla in tempo reale a chi l'abbia richiesta (colleghi, studenti, ecc.);

b) archiviare alcuni articoli utili

Esperienza diffusissima è imbattersi in articoli che si vorrebbe ricordare di aprire e leggere in un momento successivo con calma. Fra gli esperti era abitudine mandare un'e-mail con il link dell'articolo ad altri colleghi. Attualmente, è possibile condividere l'articolo interessante su Facebook, con la possibilità di renderlo disponibile solo al mondo scientifico o a una determinata lista di contatti, oppure postarlo (postare: attività di pubblicare un contenuto su un canale o una pagina on-line) su Twitter, ma in questo caso senza la facoltà di restringere il campo della visibilità a proprio piacimento. Nel caso di Twitter si rivela utile creare un account "sconosciuto" a tutti, meno che all'utente stesso, e quindi utilizzarlo come una sorta di agenda personale, dove verranno postati tutti gli articoli che sono stati "appuntati” per una successiva consultazione;

c) permettere a tutti di seguire conferenze e presentazioni in differita

È ormai abbastanza noto a tutti come sia possibile seguire una conferenza in diretta. Il sito web della SIN ha da tempo inserito i websimposia come strumento di aggiornamento professionale. Tale sistema è molto utile perché permette di seguire in diretta le presentazioni, seguendole dal vivo on-line o in differita. $\mathrm{Su}$ Youtube è possibile seguire in differita le lezioni di nefrologia come, per esempio, quelle di www.nephrologyondemand.com. Oltre che sul sito SIN è possibile seguire delle lezioni in differita anche su NDT educational e $H D C N$, tuttavia questi ultimi non offrono la possibilità di interagire commentando $o$ ponendo domande. Invece è proprio questa la prerogativa e il vantaggio stesso del web 2.0. Un'altra possibilità è quella di pubblicare i contenuti di convegni e di lezioni attraverso slide sul web, distribuendo agli internauti i nostri aggionnmenti. Un buon sistema è quello di pubblicare tali slide su siti che le ospitano (per esempio Slideshare), facendo scegliere all'Autore di renderle scaricabili o meno, ma comunque di renderle visibili a tutto schermo come se fossero proiettate nel computer dell'ospite. Anche in questo caso, il link alle slide può essere condiviso tramite pulsanti adatti (2).

\section{d) insegnare ai pazienti e/o informarli}

Il web 2.0 offre una formidabile opportunità ai sanitari (medici, infermieri, dietisti, ecc.) di accompagnare la visita con materiale informativo e di far visionare al paziente più e più volte il materiale comodamente da casa, appuntando domande e dubbi da esplicitare in un secondo momento al sanitario. Tale sistema permette di ridurre $\mathrm{i}$ tempi della visita e di standardizzare le cose da dire al paziente (i filmati potrebbero essere prodotti insieme agli infermieri e ai dietisti in modo da usare un linguaggio comprensibile chiaro e condiviso). La "Mayo Clinic" ha istituito una sezione della propria amministrazione chiamata "The Mayo Clinic Center for Social Media" appunto con lo scopo di produrre del materiale educativo per i pazienti. Suggestivo, anche se non di carattere nefrologico, è il filmato "Known Your Number", che è possibile consultare su Youtube in versione originale (3) o con la traduzione in italiano. $\mathrm{Si}$ moltiplicano iniziative istituzionali e non che sfruttano il web 2.0 attraverso messaggi di natura sociale, come la riduzione dell'abitudine al fumo, o ancora per il controllo dell'ipertensione o per migliorare l'aderenza alla terapia. In Gran Bretagna si utilizza attualmente il web 2.0 anche per campagne di sensibilizzazione sulla necessità di bere, incoraggiando la popolazione ad assumere una quantità d'acqua sufficiente a soddisfare le esigenze biologiche;

\section{e) tenersi aggiornati continuamente}

Chiunque abbia qualche anno alle spalle ben conosce la fatica di tenersi aggiornato. Tanti anni fa si andava in biblioteca per leggere almeno l'indice delle riviste; a distanza di molti anni gli indici delle riviste seguite arrivavano nelle e-mail dei professionisti con un risultato ancora poco efficiente. Le caselle postali erano limitate, le riviste erano numerose e ancora di più erano numerosi gli indici, spesso settimanali, generando alla fine una confusione insostenibile. Anche in questo aspetto il web 2.0 viene in aiuto al mondo scientifico. RSS rappresenta un buon "reader", come il rimpianto "google reader" o "iTunes", i quali sono in grado di aiutare a mettere ordine nelle varie riviste e negli indici e che, addirittura, permettono il collegamento a PubMed.

Un'ultima citazione merita il caso di iTunes U. L'App iTunes $U$ o il collegamento ad esso offrono un mondo inesplorato con una vastità di argomenti trattati. Apple, infatti, convoglia in questo sito scientifico (la $U$ di iTunes $U$ indica il termine Università) una miriade di lezioni, comunicazioni e corsi in moltissime lingue a disposizione di chi voglia aggiornarsi. Facendo un esempio, le lezioni dell'Università Federico II di Napoli sono totalmente fruibili on-line (FEDERICA) (4). Segnalare questa iniziativa ha importanza, non solo perché gratuita, ma soprattutto perché è icona di una diffusione democratica del sapere medico grazie al web, al solo costo di un $p c$ o tablet e di una connessione a Internet. Non a caso il motto Apple per iTunes $U$ è: "Learn anything, anywhere, anytime";

\section{f) formule e calcolatori}

Un tempo si portavano nella tasca del camice una fida calcolatrice e un blocco con le formule. Esperienza quotidiana per i nefrologi era avere a che fare con formule per il calcolo del filtrato, dell'escrezione frazionale di sodio, del BMI e tanto altro. Ebbene, ora il web 2.0 va incontro a tutte queste esigenze attraverso app apposite (sia Apple che Android), che gli esperti possono scaricare sul proprio tablet o, ancora meglio, sul proprio smartphone. Con orgoglio non resta che annoverare a tal proposito (purtroppo attualmente solo per Android) il pacchetto a pagamento sviluppato dal nefrologo Baracca con le più comuni formule per la nefrologia (5) e altre app aggiuntive come Medcalc, Epocrattes, Qx calculate e così via. Applicazioni e calcolatori che, soprattutto ultimamente, hanno portato enormi vantaggi per la loro completezza anche fatti da ricercatori italiani come il Dr. Barracca di Cagliari (6). 


\section{Conclusioni}

Le esperienze maturate in Italia e l'uso sempre più frequente delle nuove tecnologie 2.0 anche tra i nostri medici fanno sì che tali strumenti, attraverso un continuo confronto e una fattiva collaborazione tra colleghi, possano trovare spazio tra quelli usati tradizionalmente per lo studio e l'aggiornamento professionale.

Il percorso non è scevro di ostacoli, a cominciare dalla generale diffidenza verso le novità, soprattutto quelle tecnologiche, che condizionano le scelte di molti medici, in particolare di quelli meno giovani. Nonostante questo, la strada sembra segnata, come dimostrano i nuovi servizi basati sul concetto di "social", dove chi è impegnato nella produzione e nella diffusione dell'informazione medico-scientifica (editoria, organizzazioni istituzionali, società scientifiche) mette continuamente a disposizione degli utenti e dei lettori. D’altra parte, il nuovo generalmente fatica a imporsi, ma alla fine la storia insegna che spesso ci riesce.

Sono sul tappeto, allora, nuove questioni da analizzare e approfondire e nuove sfide da vincere. E cosa si prospetta per la medicina con il web del futuro che è alle porte? La classe medica è quella che avverte maggiormente la necessità di un ordine controllato e maturo dei contenuti che viaggiano in rete e la soluzione potrebbe essere proprio nel web 3.0.

\section{Riassunto}

Il web 2.0 e i nuovi media stanno progressivamente facendo la loro comparsa in campo medico. Da più parti i feed $R S S$, i podcast, i blog, i wiki, i social network, le on-line community e i social media, grazie alla loro semplicità d'uso e alla rapidità con la quale si stanno diffondendo, vengono indicati come strumenti innovativi per la formazione di medici, operatori sanitari, infermieri e studenti di medicina e per il loro continuo aggiornamento. L'articolo illustra le applicazioni dei principali strumenti del web 2.0 e dei social media in nefrologia e come tali strumenti stiano trasformando il modo di aggiornarsi in rete dei medici, in particolare nefrologi, e dei sanitari tutti, oltre a sottolineare come la comunicazione stia diventando sempre più un sistema di management integrato.

Parole chiave: Web 2.0, Blog, Facebook, Management, Health 2.0, Social media, Social network, Twitter, Web 2.0, Youtube

Dichiarazione di conflitto di interessi: Gli Autori dichiarano di non avere conflitto di interessi.

Contributi economici agli Autori: Gli Autori dichiarano di non aver ricevuto sponsorizzazioni economiche per la preparazione dell'articolo.

Indirizzo degli Autori:

Prof. Giuseppe Quintaliani

Via Maturanzio 31

06124 Perugia

quintaliani@yahoo.it

\section{Bibliografia}

1. http://www.slideshare.net/pqr9ap/iperpotassiemia-canavese

2. http://www.slideshare.net/pqr9ap/presentations

3. Know Your Numbers - 8675309 Parody - Mayo Clinic https:// www.youtube.com/watch? $=$ =kkps 4 XwvxK4
4. http://www.federica.unina.it/

5. http://nuke.linad.net/

6. https://play.google.com/store/apps/details?id=it.abcgo. nephrologypack 